\title{
Versatile Surface Tension and Adhesion for SPH Fluids
}

\author{
Nadir Akinci \\ University of Freiburg \\ Gizem Akinci \\ University of Freiburg \\ Matthias Teschner \\ University of Freiburg
}
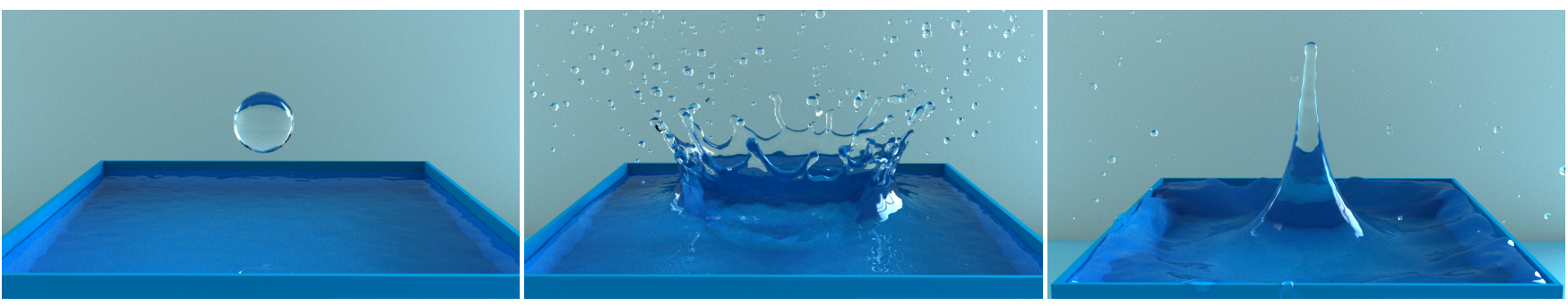

Figure 1: A water crown emerges as a result of the impact of a water droplet into a filled container. Our surface tension force allows realistic simulation of such natural phenomena.

\begin{abstract}
Realistic handling of fluid-air and fluid-solid interfaces in SPH is a challenging problem. The main reason is that some important physical phenomena such as surface tension and adhesion emerge as a result of inter-molecular forces in a microscopic scale. This is different from scalar fields such as fluid pressure, which can be plausibly evaluated on a macroscopic scale using particles. Although there exist techniques to address this problem for some specific simulation scenarios, there does not yet exist a general approach to reproduce the variety of effects that emerge in reality from fluidair and fluid-solid interactions. In order to address this problem, we present a new surface tension force and a new adhesion force. Different from the existing work, our surface tension force can handle large surface tensions in a realistic way. This property lets our approach handle challenging real scenarios, such as water crown formation, various types of fluid-solid interactions, and even droplet simulations. Furthermore, it prevents particle clustering at the free surface where inter-particle pressure forces are incorrect. Our adhesion force allows plausible two-way attraction of fluids and solids and can be used to model different wetting conditions. By using our forces, modeling surface tension and adhesion effects do not require involved techniques such as generating a ghost air phase or surface tracking. The forces are applied to the neighboring fluidfluid and fluid-boundary particle pairs in a symmetric way, which satisfies momentum conservation. We demonstrate that combining both forces allows simulating a variety of interesting effects in a plausible way.
\end{abstract}

CR Categories: I.3.7 [Computer Graphics]: Three-Dimensional Graphics and Realism-Animation;

Keywords: physically-based animation, fluid simulation, Smoothed Particle Hydrodynamics, surface tension, adhesion

Links: DD 国PDF ๑VIDEO

\section{Introduction}

Surface tension is a ubiquitous effect in daily life. For instance, when pouring water into a glass, the force that keeps liquid molecules together is the surface tension force. It is caused by cohesive forces among neighboring fluid molecules. Inside the fluid, each molecule is pulled equally by its neighbors, resulting in a zero net force. However, as the free surface does not have neighbors on all sides, the molecules in such regions are pulled inwards. Furthermore, surface tension minimizes surface area according to Laplace's law, which causes droplets of water to form a sphere when external forces are excluded. Another effect that is again caused by molecular interaction is adhesion. Adhesion allows fluids to get attracted by other materials. For instance, the unique appearance of dew on plants and the ability of water striders to stay atop water are caused by the interplay of surface tension and adhesion forces. In this paper, we focus on simulating those two molecular interaction related phenomena in the context of computer animation, more specifically for SPH (Smoothed Particle Hydrodynamics) fluids.

One important issue that arises at fluid-air and fluid-solid interfaces in SPH is density underestimation, where densities of the particles are erroneously computed as less than the rest density when the density summation approach is used. Those wrong density values result in negative pressures and cause the particles to cluster, which is known as tensile instability in SPH. This phenomenon can be alleviated by using artificial pressure forces [Monaghan 2000; Macklin and Mueller 2013], which, however, result in spurious surface tensions. For this reason, either a density correction technique [Shepard 1968], or simply not allowing negative pressures are other common practices for avoiding tensile instability. However, this still does not solve the problem of sticking particles at the fluid interface, since the pressure field is still not reconstructed in a physically sensible way. [Akinci et al. 2012b] addresses this issue for fluid-solid interface by pre-computing a single layer of boundary particles for the solid boundaries, which also extends to two-way fluid-solid coupling. In [Schechter and Bridson 2012], ghost SPH particles are dynamically generated at both fluid-solid and fluid-air interfaces.

For modeling surface tension in SPH, additional techniques are generally preferred. These can be listed as: Curvature based external forces on particles (e.g. [Müller et al. 2003]), pairwise forces based on cohesion (e.g. [Becker and Teschner 2007]), a modified SPH formulation [Clavet et al. 2005], and more recently forces based on surface mesh curvature [Yu et al. 2012]. However, there is no sin- 
gle approach, which can handle very large surface tensions, avoids particle clustering at the free surface, minimizes surface curvature, and conserves momentum at the same time. Adhesion of fluids to solids is modeled in SPH by using a distance based attraction force [Clavet et al. 2005] and by using the combination of ghost solid particles and XSPH [Schechter and Bridson 2012] between the fluid and the solid boundary. However, neither of the methods is capable of simulating some important real world scenarios such as different wetting effects. Furthermore, the effect of adhesion forces on the solids is neglected in both works. Our surface tension and adhesion schemes are the first to meet all these requirements, and allow plausible simulations of variety of effects that emerge in reality. Additionally, our surface tension force avoids particle clustering at the free surface without generating ghost air particles using [Schechter and Bridson 2012] or artificial pressure forces [Monaghan 2000]. In the remainder of this section, we first discuss existing works that model surface tension and adhesion effects in fluid animation with an emphasis on SPH fluids, and then we highlight the benefits of our techniques in comparison to the existing work.

\subsection{Surface Tension in Fluid Simulation}

As surface tension has a quite significant role in the appearance of liquids, many researchers have investigated techniques for incorporating surface tension to fluid simulations. In the context of $\mathrm{SPH}$, the early surface tension techniques focus on applying forces to minimize surface curvature [Morris 1999; Müller et al. 2003]. Those approaches compute normals for each particle, which determine the direction of the force and can be computed using the gradient of the smoothed color field. The magnitude of the force is based on the curvature at a particle position, which can be computed by taking the second derivative of the smoothed color field, or the divergence of the normal field. However, there exist important issues with such techniques. First of all, for the particles that are inside of the fluid, normalizing the smoothed color gradient can result in arbitrary normals for inner particles. This problem has been avoided by applying the surface tension force to the particles whose smoothed color gradient has a magnitude larger than a threshold value (which however results in discontinuous forces). The second problem is that curvature estimation is very sensitive to particle disorder in SPH because of requiring the second derivative. The third problem with those techniques is that the forces are applied to the fluid particles as external forces in a non-symmetric way, which invalidates momentum conservation.

Because of the important limitations of the approaches that are based on surface curvature and normal information, researchers proposed new techniques to address the surface tension problem on a molecular level by using cohesion forces between neighboring fluid particles [Tartakovsky and Meakin 2005; Becker and Teschner 2007]. Therefore, these techniques avoid both normal computation and the erroneous curvature estimation. Another benefit of these techniques is that they trivially conserve momentum as the applied forces are pairwise symmetric. However, only using cohesion forces between fluid particles does not guarantee surface area minimization as the forces can trivially balance each other in a form that does not necessarily correspond to the smallest surface area. As we will show in our paper, large cohesion forces between particles can also result in unrealistic fluid patterns, such as cobweb-like elongating fluid structures. For large surface tensions, such structures do not easily break as using attraction forces alone does not minimize surface area of the fluid, but strengthens the already existing structures. Another approach that applies attraction forces between neighboring fluid particles to generate surface tension is [Clavet et al. 2005]. In this work, the basic SPH scheme is reformulated by using double density relaxation, where the surface tension force is computed based on the negative pressures that arise at the free surface similar to [Monaghan 2000]. However, as the surface tension arises as a side effect, different surface tension behaviors cannot be modeled with this work. Furthermore, this work also does not take surface area minimization into account.

More recently, [Yu et al. 2012] proposed another solution to the surface tension problem in SPH. In this work, the curvature is estimated on the fluid surface mesh, but the generated surface tension forces are applied to the adjacent fluid particles enclosed by the mesh. They show that when the surface mesh has more samples than the fluid (i.e. more vertices than the particles); the curvature computation is not as error prone as it is when computing curvature from the particles. Furthermore, a nice comparison of the method to [Becker and Teschner 2007] is given in the paper for the experiment where a cubic droplet deforms to a sphere. However, there exist some limitations of the technique. First of all, the employed surface tracking scheme may fail to detect isolated fluid pieces in regions where the mesh resolution remains coarse; this prevents generating surface tension for such areas. Furthermore, the quality of the surface tension depends on the tracked mesh resolution. Finally, requiring an explicit representation of the fluid surface in each simulation step is an overhead for the cases where an explicit surface is not required (e.g., for interactive scenarios such as [Macklin and Mueller 2013], or when an efficient view dependent surface reconstruction scheme is preferred [Fraedrich et al. 2010]).

In the context of grid based fluids, there exist many different techniques for incorporating surface tension: e.g., from a level set function [Kang et al. 2000], by employing an octree structure for more accurate force evaluation [Losasso et al. 2004], by treating surface tension as discontinuous boundary conditions [Hong and Kim 2005], based on surface energy [Misztal et al. 2010; Batty et al. 2012], and based on the surface mesh [Brochu et al. 2010; Thürey et al. 2010]. As we focus on fully Lagrangian flow, a detailed discussion of these works is beyond the scope of our paper.

\subsection{Solid Adhesion in Fluid Simulation}

[Steele et al. 2004] proposed a fully Lagrangian approach for the simulation of viscous liquids, including adhesion to solids. They define adhesion properties of different types of materials using distance-dependent forces. However, the employed linear density kernel and strict anti-penetration constraints limit their approach to highly viscous liquids. Later in [Clavet et al. 2005], adhesion of viscoelastic SPH fluids to solids is modeled by using a distance based attractive force term, which is added as an impulse to the fluid particles. The authors demonstrated interesting scenarios such as droplet formation and sticking of fluids to solids. More recently in [Schechter and Bridson 2012], fluid to solid adhesion is accomplished by computing a ghost velocity at each solid particle by combining solid's own velocity and the tangential component of the nearest fluid particle's velocity. After this step, fluid to solid adhesion is generated using an XSPH based artificial viscosity term. In [He et al. 2012], sticking of fluid particles to solids arises as a side effect of the employed velocity constraints to realize different slip conditions, which makes it difficult to model different adhesion related effects.

In the context of Eulerian approaches, adhesion of fluids to solids can be accomplished by adjusting the velocity or pressure constraints enforced along the boundaries (e.g. [Goktekin et al. 2004]). In addition to the general techniques, adhesion of fluids is modeled to animate variety of interesting scenarios such as sticking of viscous threads [Bergou et al. 2010] and sheets [Batty et al. 2012] to solids, wetting of hair [Rungjiratananon et al. 2012], animating droplets on a glass surface [Chen et al. 2012] and sintering of snow [Takahashi and Fujishiro 2012]. 

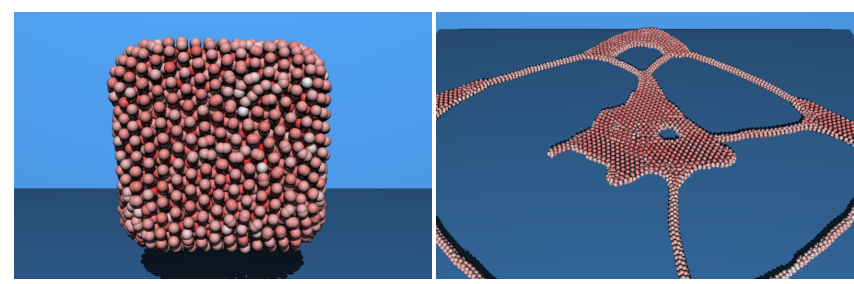

(a) [Tartakovsky and Meakin 2005]
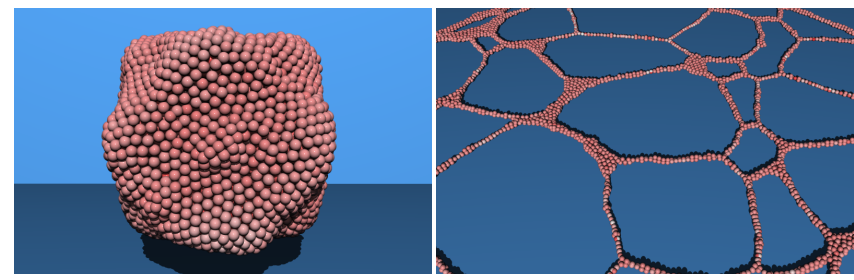

(b) [Becker and Teschner 2007]
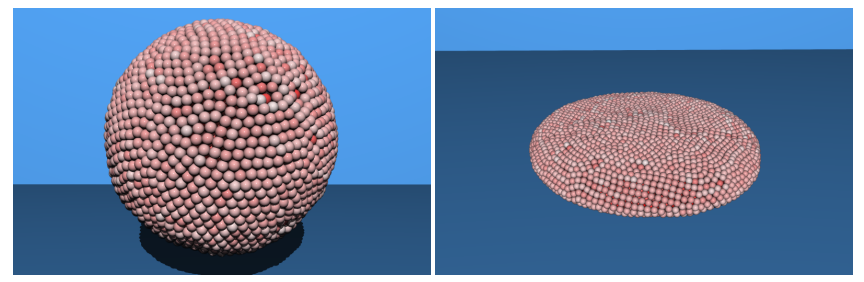

(c) Our surface tension model.

Figure 2: A fluid droplet in the shape of a cube is left to transform to a sphere and then dropped on the ground. Particle clustering and non-uniform particle alignment is visible in both $(a)$ and $(b)$, which is addressed with our surface tension model (c). Furthermore, neither of the cohesion-only models are able to simulate the large surface tension possible with our model, but they result in cobweb-like elongating structures, no matter how large the cohesion forces are. Particles are colored according to pressure.

\subsection{Contributions}

We present a surface tension force and a fluid-solid adhesion force for the improved treatment of fluid-air and fluid-solid interfaces in SPH fluids. Our surface tension approach can handle large surface tensions by minimizing surface area in all scales while conserving momentum. Furthermore, our surface tension force also generates repulsion forces for close distances, which prevents the particleclustering problem at the free surface without requiring additional treatment such as generating ghost air particles or artificial pressure forces. Our adhesion force allows physically plausible solid-fluid adhesion effects without requiring additional handling such as ghost SPH. Furthermore, our approach allows simulating interesting phenomena such as different wetting effects and two-way adhesion. Both of our forces can be easily added to an existing SPH solver as additional force terms without any extra effort. By combining our surface tension and adhesion forces, we are able to simulate a variety of effects that can be observed in nature.

\section{Surface Tension Model}

Surface tension in liquids arises as a result of molecular cohesion. However, as SPH simulates fluids on a macroscopic level with a finite support radius $h$, cohesion forces between SPH particles do not reproduce the surface tension that we observe in reality. When only cohesion forces are applied between particles, the neighboring particles just attract each other which can result in any arbitrary

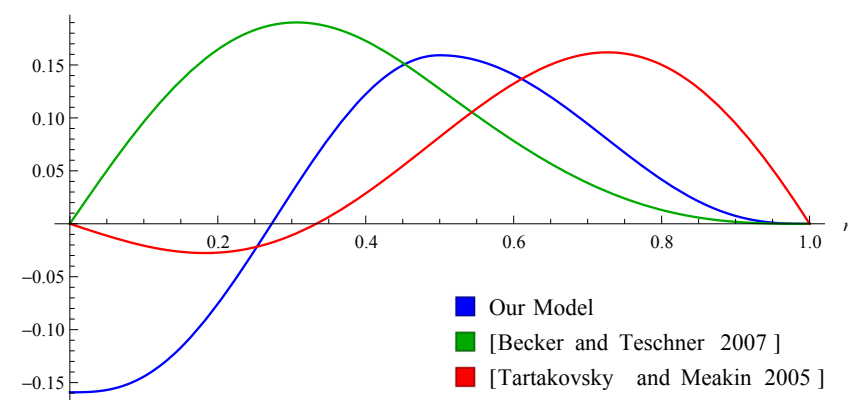

Figure 3: Comparison of the shape of our cohesion force (blue) to [Tartakovsky and Meakin 2005] (red) and [Becker and Teschner 2007] (green) inside the SPH support radius $h=1$.

configuration depending on the initial configuration of the particles (see e.g. Figures $2 \mathrm{a}, 2 \mathrm{~b}$ ). Therefore, surface area minimization is not guaranteed. Additionally, we also experienced that only using curvature minimization terms [Morris 1999; Müller et al. 2003] results in more severe particle clustering for the experiment depicted in Figure 2, causing the droplet to break into many smaller droplets. To address these issues, we propose a new surface tension force that takes both molecular cohesion and surface area minimization into account and allows handling large surface tensions properly (see Figure 2c).

\subsection{Cohesion Term}

[Tartakovsky and Meakin 2005] is the first work to employ molecular cohesion forces to generate surface tension in SPH. They adjusted the cosine function to generate attraction for distant particles, and repulsion for close particles. However, we observed that the function used in [Tartakovsky and Meakin 2005] results in clustering throughout the fluid (see Figure 2a). Later in [Becker and Teschner 2007], instead of the cosine function, the SPH kernel function is used. However, since the method lacks a repulsion term, it also results in severe clustering; especially in regions with underestimated pressures (see Figure 2b-right). Both methods are applied through the displacement vector between the neighboring particles. This causes the forces to vanish for very close neighbors, which is another reason why clustering occurs with these methods. The forces applied by those two methods for a support radius $h=1$ can be seen in Figure 3.

Because of the issues with the existing cohesion forces, we propose an alternative cohesion force defined as:

$$
\mathbf{F}_{i \leftarrow j}^{\text {cohesion }}=-\gamma m_{i} m_{j} C\left(\left|\mathbf{x}_{i}-\mathbf{x}_{j}\right|\right) \frac{\mathbf{x}_{i}-\mathbf{x}_{j}}{\left|\mathbf{x}_{i}-\mathbf{x}_{j}\right|}
$$

where $i$ and $j$ are neighboring fluid particles, $m$ denotes mass and $\mathbf{x}$ denotes position of the respective particle, $\gamma$ is the surface tension coefficient and $C$ is a spline function that we created for a 3D SPH simulation as:

$$
C(r)=\frac{32}{\pi h^{9}} \begin{cases}(h-r)^{3} r^{3} & 2 r>h \wedge r \leq h \\ 2(h-r)^{3} r^{3}-\frac{h^{6}}{64} & r>0 \wedge 2 r \leq h \\ 0 & \text { otherwise }\end{cases}
$$

The term $h^{9}$ in the denominator of (2) is a normalization factor to make the force result in the same acceleration for different support radii (e.g. like the SPH pressure force). The constant term in the beginning of (2) is basically used to shift the practical $\gamma$ values close to 1. Similar to [Tartakovsky and Meakin 2005], our cohesion force also has both a positive and a negative part to result in repulsion (see 
Figure 3). For the attraction term, we chose a maximum around the particle rest distance $h / 2$, where the attraction smoothly vanishes to 0 until the support radius $h$. For fluid neighbors closer than the rest distance, the force smoothly decreases to a negative value, which results in repulsion forces for the particles that are too close to each other. Furthermore, both the attraction and repulsion forces behave like a Gaussian to avoid clustering, which is in contrast to [Tartakovsky and Meakin 2005]. Additionally, our repulsion force does not vanish to 0 for close neighbors, which prevents particle clustering in regions with underestimated pressures (see Figure 2c). Our cohesion term also has similarities to the Lennard-Jones potential [Jones 1924], which is a commonly used model to approximate the interaction between a pair of molecules. However, the main difference of our cohesion term to the Lennard-Jones potential is that our term stops increasing as the particles move closer, which helps to avoid too stiff forces and resultant stability issues.

\subsection{Surface Area Minimization Term}

Although our cohesion force solves some important issues of previous cohesion models, it is still not sufficient for minimizing the fluid surface area because of the reasons we discussed previously. Therefore, we use an additional force term to counteract surface curvature to minimize the surface area. Since computing curvature from particles is error-prone in SPH, different from the previous models, we avoid computing surface curvature explicitly. We firstly compute normal information by applying the SPH approximation to the gradient of the smoothed color field as:

$$
\mathbf{n}_{i}=h \sum_{j} \frac{m_{j}}{\rho_{j}} \nabla W\left(\left|\mathbf{x}_{i}-\mathbf{x}_{j}\right|\right)
$$

where $W$ is the SPH kernel function and $\rho_{j}$ is neighboring particle density. Different from [Morris 1999] and [Müller et al. 2003], we have the factor $h$ to make the computed normal scale independent. At this point, we use the fact that the magnitude of $\mathbf{n}_{i}$ is proportional to the curvature, where its value is close to zero for the inner parts of the fluid, but large at the free surface proportional to the curvature. We utilize this information and finally create a symmetric force as:

$$
\mathbf{F}_{i \leftarrow j}^{\text {curvature }}=-\gamma m_{i}\left(\mathbf{n}_{i}-\mathbf{n}_{j}\right) .
$$

One can easily confirm that (3) is zero in flat regions (as $\mathbf{n}_{i}-\mathbf{n}_{j}=$ $\mathbf{0}$ ) and inside the fluid (as $\mathbf{n}_{i} \approx \mathbf{0}$ and $\mathbf{n}_{j} \approx \mathbf{0}$ ), but it gets larger as the curvature increases. Therefore, our surface area minimization force avoids two important issues of previous techniques: Normalization of $\mathbf{n}_{i}$ (that is erroneous inside the fluid), and explicit curvature computation (that is very sensitive to particle disorder).

\subsection{Combined Surface Tension Force}

Before discussing the combined surface tension force, we will discuss another important particle deficiency related issue in SPH. For the fluids in reality, attractions between fluid molecules occur at a microscopic scale, where each molecule interacts with many other molecules. In SPH, however, particles represent macroscopic volumes of the fluid. If we consider the simple example of two neighboring particles, the net attraction force that affects each particle is smaller than in a configuration of three neighboring particles, which makes the two particles separate easier than the three particles in the case of external forces. If those macroscopic particles would have been sampled with real water molecules, there would not be such a difference. This error in SPH manifests itself as too many isolated particles, since particles with smaller neighborhood get isolated easier than the rest of the fluid. There exist techniques to address this issue, such as corrected SPH (e.g. [Belytschko et al. 1998])

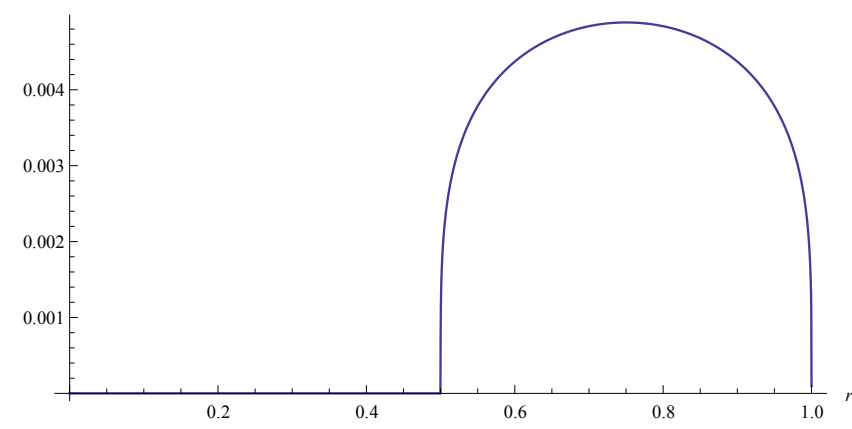

Figure 4: The shape of our adhesion function inside the SPH support radius $h=1$.

and adaptive SPH [Shapiro et al. 1996]. However, these works add too much computational overhead to basic SPH. Although [Monaghan 2000] (also used in [Macklin and Mueller 2013]) implicitly addresses this issue by generating a spurious surface tension, such an approach is not desirable for our purposes since it would interfere with our refined surface tension model. We provide an explicit solution to this problem by creating the following symmetrized correction factor:

$$
K_{i j}=\frac{2 \rho_{0}}{\rho_{i}+\rho_{j}},
$$

where $\rho_{0}$ is the rest density of the fluid, and $\rho_{i}$ and $\rho_{j}$ are the densities of the neighboring fluid particles. As we do not correct the fluid particle densities, a fluid particle with less than full neighborhood has $K_{i j}>1$, and a fluid particle with full neighborhood has $K_{i j} \approx 1$. Therefore, $K_{i j}$ amplifies forces for the particles with neighborhood deficiency, while the forces remain the same for the particles with appropriate neighborhood. The final surface tension force can be written as:

$$
\mathbf{F}_{i \leftarrow j}^{s t}=K_{i j}\left(\mathbf{F}_{i \leftarrow j}^{\text {cohesion }}+\mathbf{F}_{i \leftarrow j}^{\text {curvature }}\right) .
$$

Note that the terms in (5) are fully symmetrized, and the total force is applied to the particle pairs. This is, however, not the case in previous surface area minimization techniques (e.g. [Morris 1999; Müller et al. 2003; Yu et al. 2012]), as they apply the forces to the particles as external forces. The effect of combining both terms can be seen in Figure 2c. Note the large surface tension possible with our approach. Although we used larger cohesion forces for both [Tartakovsky and Meakin 2005] and [Becker and Teschner 2007], they failed to generate the large surface tension that we wanted to achieve. We also observed that using larger surface tension forces with the cohesion-only models does not further improve the quality of the generated surface tension behavior, but only results in stiffer fluids with similar spurious structures.

\section{Adhesion Model}

Different from cohesion, adhesion occurs as a result of molecular interaction of dissimilar materials. In our work, we focus on twoway fluid-solid adhesion in SPH simulations.

For boundary handling and two-way fluid-solid coupling, we use [Akinci et al. 2012b], where solid surfaces are sampled using boundary particles. In this approach, the volume of a boundary particle is approximated as $V_{b_{i}}=\frac{1}{\delta_{b_{i}}}$, where $\delta_{b_{i}}$ is the number density of a boundary particle computed according to the neighboring boundary particles. The contribution of a boundary particle to a fluid particle (and vice versa) is based on the volume of a boundary particle and written as $\Psi_{b_{i}}\left(\rho_{0}\right)=\rho_{0} V_{b_{i}}$, where $\Psi_{b_{i}}$ is 
used in place of the mass of a boundary particle when computing fluid density, pressure forces and viscosity forces. Therefore, the approach addresses the sticking problem of SPH near solid boundaries, and allows two-way fluid-solid coupling with different slip conditions. Adhesion effects, however, are not addressed in [Akinci et al. 2012b].

In our work, we compute adhesion forces between the fluid and the boundary particles as:

$$
\mathbf{F}_{i \leftarrow k}^{a d h e s i o n}=-\beta m_{i} \Psi_{b_{k}} A\left(\left|\mathbf{x}_{i}-\mathbf{x}_{k}\right|\right) \frac{\mathbf{x}_{i}-\mathbf{x}_{k}}{\left|\mathbf{x}_{i}-\mathbf{x}_{k}\right|}
$$

where $k$ denotes boundary particles, $\mathbf{x}$ denotes position of the respective particle, $\beta$ is the adhesion coefficient and $A$ is a spline function that we created for a $3 \mathrm{D} \mathrm{SPH}$ simulation as:

$$
A(r)=\frac{0.007}{h^{3.25}} \begin{cases}\sqrt[4]{-\frac{4 r^{2}}{h}+6 r-2 h} & 2 r>h \wedge r \leq h \\ 0 & \text { otherwise }\end{cases}
$$

Similar to our cohesion force, the term $h^{3.25}$ in the denominator of (7) is a normalization factor to make the force result in the same acceleration for different support radii. The scalar term in front of (7) is used to be able to select $\beta$ values in the similar range of $\gamma$ values, where $\beta \approx \gamma$ models a moderate hydrophilic behavior. Since the boundary forces used in [Akinci et al. 2012b] already prevent clustering near the solid boundaries, we designed our adhesion force to only attract particles with distances between $h / 2$ and $h$. Furthermore, we tried to make our attraction force large in this interval, while keeping the force continuous (see Figure 4). Initially, we started with a Gaussian-like shape for the adhesion force. However, such a force was causing most of the fluid (except the closest fluid layer) to unrealistically detach from the solid, regardless of the magnitude of the adhesion force. Finally, we came up with such a steep parabolic function, which generates strong attractions for most of the neighboring fluid particles.

Note that similar to our surface tension force, our adhesion force is also fully symmetric, where $\mathbf{F}_{k \leftarrow i}^{\text {adhesion }}=-\mathbf{F}_{i \leftarrow k}^{\text {adhesion }}$. Our adhesion force allows simulating interesting scenarios, such as different wetting conditions and two-way adhesion.

\section{Implementation}

We use [Solenthaler and Pajarola 2009] for computing SPH pressures. However, our surface tension and adhesion forces can be integrated into any SPH solver (e.g. [Becker and Teschner 2007; Bodin et al. 2011; Macklin and Mueller 2013; Ihmsen et al. 2013]) since the forces are computed from the particles and are directly applied to the neighboring pairs. Furthermore, we do not apply pressure forces that arise from negative pressures. For time-step selection, we use the adaptive scheme in [Ihmsen et al. 2010]. For boundary handling and two-way solid-fluid coupling, we employ [Akinci et al. 2012b] and simulate dynamic objects using Bullet [Coumans 2011]. We use the artificial viscosity model described in [Monaghan 2005] both for fluid-fluid and fluid-solid interactions as done in [Akinci et al. 2012b]. Because of the particle deficiency related reason highlighted in Section 2.3, we also multiply viscosity forces with (4). We use the cubic spline kernel function [Monaghan 2005] for our SPH simulations. For SPH neighborhood search, we use the compact hashing technique explained in [Ihmsen et al. 2011b].

If not stated otherwise, we used $\gamma=1$ for all experiments to mimic the surface tension of water. Furthermore, we used $\beta=\gamma$ to model moderate hydrophilic interactions. In all of our simulations, we kept fluid compressibility below $0.1 \%$. Depending on the scale of
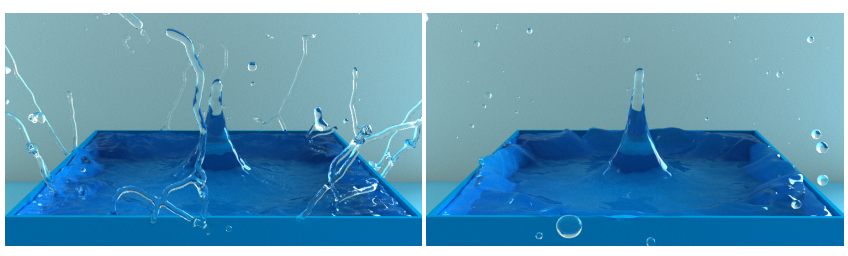

Figure 5: Comparison of a selected frame from the water crown experiment using [Becker and Teschner 2007] (left) and our surface tension model (right). Note the spurious surface tension and bumpy fluid surface in the left image. Such effects are avoided with our approach as the fluid surface area is properly minimized.

the simulated setting, we used different particle radii $r$, where the particle spacings and support radii were $2 r$ and $4 r$ respectively. We used very low artificial viscosity for the simulated fluids $(\sim 0.01)$ to mimic the viscosity of water. If not stated otherwise, all solids in our experiments also have the same viscosity to model a moderate slip condition when interacting with the fluids. We reconstructed the fluid surfaces using the efficient implementation explained in [Akinci et al. 2012a]. The renderings were performed using mental ray [NVIDIA ARC 2011]. All simulations and renderings were run on an Intel Xeon X5690 with 16 GB RAM. For the presented scenes, the average simulation time per frame was between 0.1 to 15 seconds, depending on the complexity of the scene, where several simulations steps were computed for each frame.

\section{Results}

In this section, we demonstrate the versatility of our surface tension and adhesion models in different simulation scenarios. Our experiments show plausible fluid-fluid and fluid-solid interactions, even at the scale of a single droplet.

To show that our approach can handle realistic fluid-fluid interactions, we dropped a $6.5 \mathrm{~cm}^{3}$ fluid droplet into a $15 \times 4 \times 15 \mathrm{~cm}^{3}$ container filled with $1 \mathrm{M}$ fluid particles (Figure 1). After the fluid droplet hits the main fluid body in the container, a realistic water crown emerged. Also note how the splashes form spherical droplets with various sizes, which is a phenomenon observed in reality. Note both the thin features around the crown and the smooth fingerings that emerge at its top. Later in the simulation, a vertical finger occurred, which is an effect that occurs after a water crown collapses. With the previous surface tension models that are only based on cohesion, the experiment reveals many spurious fluid structures (see Figure 5).

Our next experiment shows that, by using our surface tension and adhesion models, it is possible to simulate the impact of a large fluid droplet on a solid object in a realistic way. A $0.5 \mathrm{~m}^{3}$ water volume consisting of $100 \mathrm{~K}$ fluid particles was dropped on a $1.5 \times 0.12 \times 1.5$ $\mathrm{m}^{3}$ solid (Figure 6). The adhesion of solid was chosen as $\beta=0.6$ to model a reduced hydrophilic behavior. Because of the surface tension, sheets of fluid broke into fingers, which then transformed into spherical droplets (Figure 6 middle-left). The adhesion of the table resulted in sliding droplets dripping from the side of the table (Figure 6 middle-right). Furthermore, the adhesion prevented the fluid to completely merge to a single water body at the end (Figure 6 right).

In the next experiment, we show how the combination of our surface tension and adhesion forces can be used to simulate a scenario where a vertical water stream realistically flows over a sphere with diameter $5 \mathrm{~cm}$ (Figure 7). There were up to $50 \mathrm{~K}$ fluid particles in the scene. We are able to simulate such vertical flows with solid 

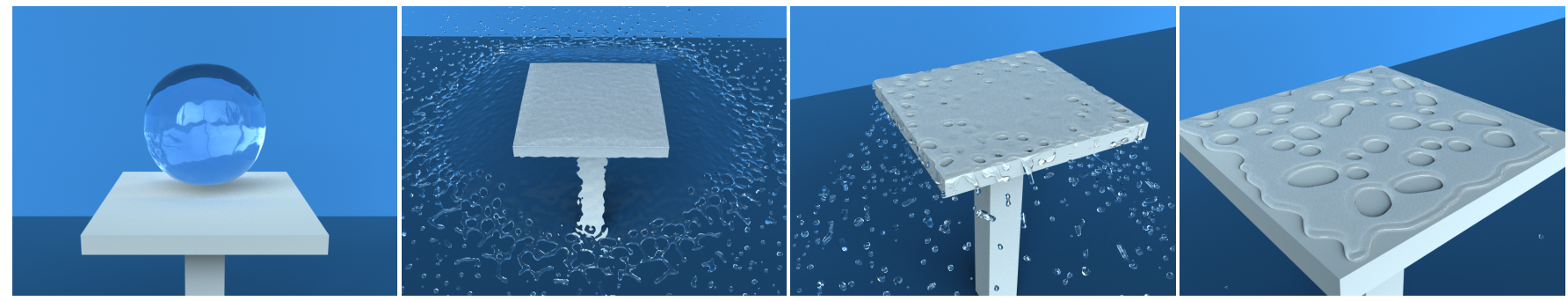

Figure 6: A spherical water volume collides with a planar solid object. The interplay of our surface tension and adhesion forces allows realistic interactions in such scenarios.
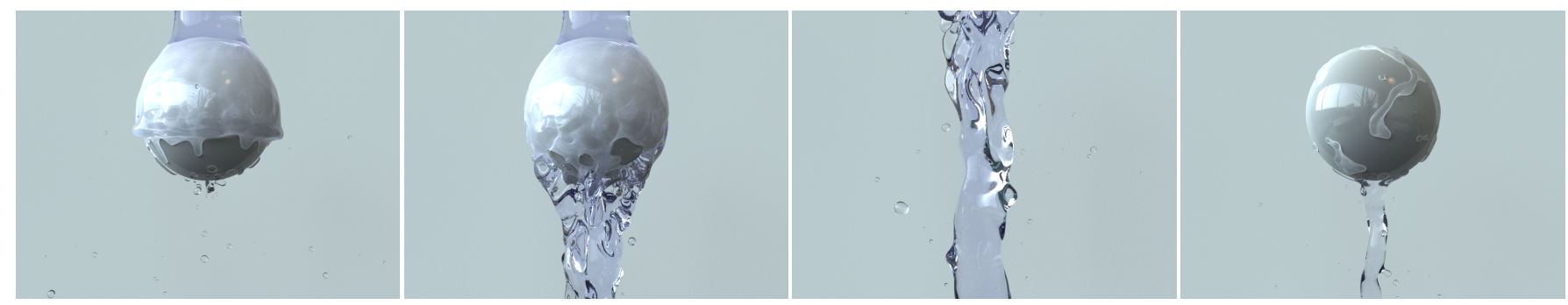

Figure 7: Pouring water on a sphere. Our forces allow simulating a realistic stream flowing over a sphere without using ghost SPH.
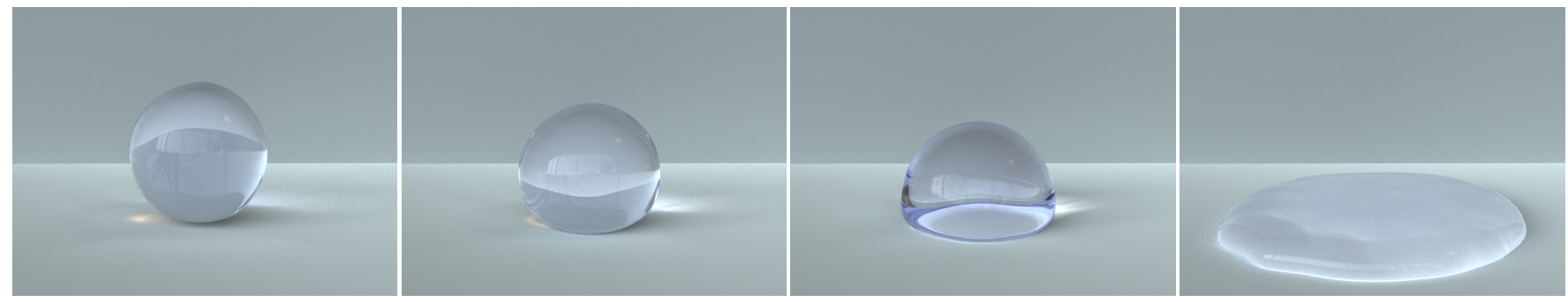

Figure 8: Combination of our surface tension and adhesion forces allows simulating different wetting effects, from no wetting (left), intermediate wetting (middle), up to perfect wetting (right), in the same simulation scale.

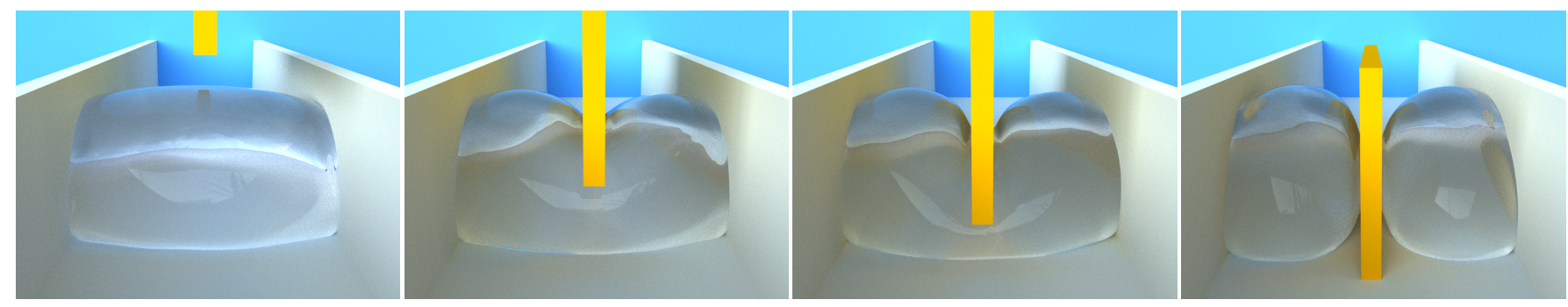

Figure 9: A droplet in an adhesive box is split into two with an object with zero adhesion. Note how the fluid sticks to the box but maintains a much smaller contact area with the splitting object because of the wetting difference.
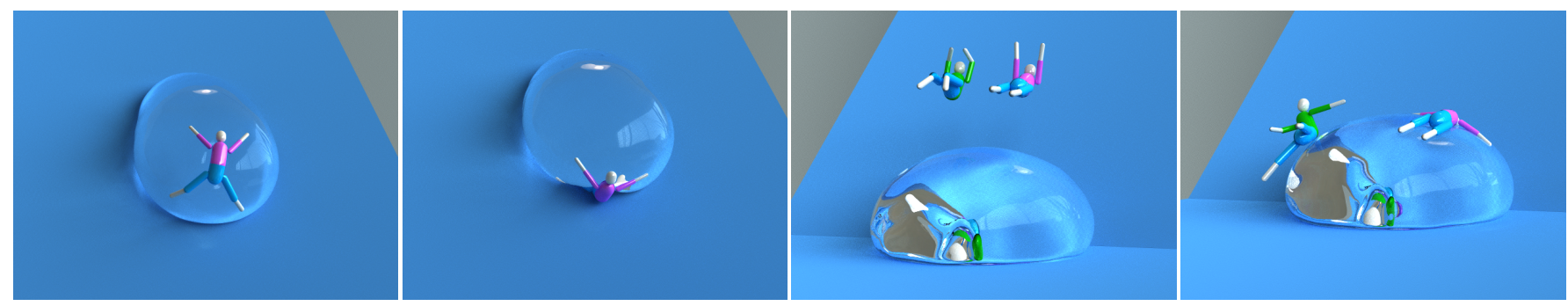

Figure 10: Using the right balance of surface tension, adhesion, and fluid-solid viscosity forces allows us to simulate rolling water droplets. In this scene, ragdolls with different adhesion properties interact differently with the droplet. 
adhesion realistically without requiring ghost $\mathrm{SPH}$.

In another experiment we show that by using different adhesion and surface tension constants (where $1 \geq \gamma, \beta \geq 0.001$ ), we were able to simulate different wetting conditions in the same simulation scale between no wetting up to perfect wetting for a $1 \mathrm{~cm}^{3}$ fluid droplet consisting of 750 particles (see Figure 8).

We created our next experiment to demonstrate how fluids react differently to two solid objects with different adhesion in the same environment (Figure 9). In this scene, a $1 \mathrm{~cm}^{3}$ water droplet consisting of $4 \mathrm{~K}$ particles was resting in a highly hydrophilic box with $\beta=2$. Afterwards, another object with zero adhesion split the droplet into two parts. Note how the droplet sticks to the box, while its contact area with the splitting object remains small.

In our final experiment, we show how two-way adhesion force can be used to create interesting scenarios (Figure 10). In this scene, we dropped a $27 \mathrm{~cm}^{3}$ fluid droplet consisting of $14 \mathrm{~K}$ particles with $\gamma=3$, on an inclined plane. The plane had $\beta=1.2$ to make the droplet create a large contact angle with the plane. Additionally, we set the viscosity of the inclined plane to 1 (which was 0.01 in the other experiments). Such a large viscosity makes the droplet roll on the surface, instead of slide. In this experiment, we also dropped ragdolls on the fluid droplet with a density two times larger than the fluid density. This experiment also shows that large surface tension forces prevent the solid to penetrate into the fluid, which is a phenomenon observed in nature. Furthermore, pink ragdolls (with $\beta=1$ ) stick to the droplet, whereas the green ragdolls (with $\beta=0$ ) bounce and slip from the droplet.

\section{Discussion and Future Work}

In the presented experiments, when the surface tension is the dominant force acting on a particle, time steps are limited by the surface tension. This was the case for some of our experiments that were performed in droplet scales. However, for most of the larger scale experiments, the pressure force was the dominant force. However, as we used the adaptive time stepping scheme explained in [Ihmsen et al. 2010] that also takes the total force on a particle into account, we did not run into stability issues when performing our experiments.

In reality, surface tension arises because of cohesion forces between fluid molecules of the same fluid phase, independent of what is beyond the free surface (e.g. air, another liquid or vacuum). Therefore, modeling surface tension does not require an explicit second fluid phase. One ubiquitous effect that arises in reality because of multi-phase interactions is air bubbles inside fluids. Since simulating density ratios in the order of $\rho_{\text {water }} / \rho_{\text {air }}$ would require considerably more computational effort, there exist alternative air bubble generation techniques to avoid multi-phase simulations (e.g. [Hong et al. 2008; Ihmsen et al. 2011a; Busaryev et al. 2012]). We believe that the realism of our results can be further improved by using such a method.

Recently, [Yu et al. 2012] demonstrated that sub-particle scale capillary waves that are directly simulated on the fluid surface mesh can add a significant amount of detail to an existing simulation. We believe that our results would further improve by using their surface tracking and capillary wave simulation approach.

\section{Conclusion}

We presented a new surface tension force and a new fluid-solid adhesion force to improve the handling of fluid-air and fluid-solid interfaces in SPH simulations. Our surface tension force allows simulating large surface tension, minimizes surface curvature, addresses the particle-clustering problem in SPH, and conserves momentum, all at the same time. Our adhesion force allows physically plausible fluid-solid adhesion effects, including symmetric adhesion, and can be used to model different surface wettings. Furthermore, our forces can be easily added to an existing SPH solver. Combining both forces allowed us to simulate a variety of interesting scenarios that have not been shown in the graphics literature yet.

\section{Acknowledgments}

We thank the reviewers for their helpful comments. This project is supported by the German Research Foundation (DFG) under contract numbers SFB/TR-8 and TE 632/1- 2. We also thank NVIDIA $\mathrm{ARC} \mathrm{GmbH}$ for supporting this work.

\section{References}

Akinci, G., Ihmsen, M., Akinci, N., And Teschner, M. 2012. Parallel surface reconstruction for particle-based fluids. Computer Graphics Forum 31, 1797-1809.

Akinci, N., IhMSEn, M., AKInCI, G., Solenthaler, B., AND TESCHNER, M. 2012. Versatile rigid-fluid coupling for incompressible SPH. ACM Trans. on Graphics (SIGGRAPH Proc.) 31, $4,62: 1-62: 8$

Batty, C., Uribe, A., Audoly, B., And Grinspun, E. 2012. Discrete viscous sheets. ACM Trans. on Graphics (SIGGRAPH Proc.) $31,4,113$.

Becker, M., And Teschner, M. 2007. Weakly compressible SPH for free surface flows. In Proc. of the 2007 ACM SIGGRAPH/Eurographics Symposium on Computer Animation, 209-217.

Belytschio, T., Kronagauz, Y., Dolbow, J., And GerLACH, C. 1998. On the completeness of meshfree particle methods. Int. J. Numer. Meth. Engng. 43, 785-819.

Bergou, M., Audoly, B., Vouga, E., Wardetzky, M., And Grinspun, E. 2010. Discrete viscous threads. ACM Trans. on Graphics 29, 4, 116.

Bodin, K., Lacoursiere, C., And Servin, M. 2011. Constraint fluid. IEEE Transactions on Visualization and Computer Graphics, 99, 1-12.

Brochu, T., BATTy, C., AND BRIDSON, R. 2010. Matching fluid simulation elements to surface geometry and topology. ACM Trans. on Graphics (SIGGRAPH Proc.) 29, 4, 47.

Busaryev, O., Dey, T., Wang, H., And Ren, Z. 2012. Animating bubble interactions in a liquid foam. ACM Trans. on Graphics (SIGGRAPH Proc.) 31, 4, 63.

Chen, K.-C., Chen, P.-S., And Wong, S.-K. 2012. A hybrid method for water droplet simulation. In Proceedings of the 11th ACM SIGGRAPH International Conference on Virtual-Reality Continuum and its Applications in Industry, ACM, 341-344.

Clavet, S., Beaudoin, P., and Poulin, P. 2005. Particlebased viscoelastic fluid simulation. In $S C A$ '05: Proceedings of the 2005 ACM SIGGRAPH/Eurographics symposium on Computer animation, ACM Press, New York, NY, USA, 219-228.

Coumans, E., 2011. Bullet physics library (version 2.78) [software]. http://www.bulletphysics.org.

Fraedrich, R., Auer, S., AND Westermann, R. 2010. Efficient high-quality volume rendering of SPH data. Visualization 
and Computer Graphics, IEEE Transactions on 16, 6, 15331540.

Goktekin, T., Bargteil, A., And O'Brien, J. 2004. A method for animating viscoelastic fluids. ACM Trans. on Graphics (SIGGRAPH Proc.), 463-468.

He, X., Liu, N., Wang, G., Zhang, F., Li, S., Shao, S., And WANG, H. 2012. Staggered meshless solid-fluid coupling. ACM Trans. on Graphics (SIGGRAPH Asia Proc.) 31, 6, 149.

Hong, J.-M., AND KIM, C.-H. 2005. Discontinuous fluids. ACM Trans. on Graphics (SIGGRAPH Proc.), 915-920.

Hong, J.-M., LeE, H.-Y., Yoon, J.-C., AND KIM, C.-H. 2008. Bubbles alive. ACM Trans. on Graphics (SIGGRAPH Proc.) 27, 48:1-48:4.

Ihmsen, M., Akinci, N., Gissler, M., And Teschner, M. 2010. Boundary handling and adaptive time-stepping for PCISPH. In Proc. of VRIPHYS, 79-88.

IhMSen, M., BADER, J., AKInCI, G., AND Teschner, M. 2011. Animation of air bubbles with SPH. In International Conference on Graphics Theory and Application, 225-234.

Ihmsen, M., Akinci, N., Becker, M., And Teschner, M. 2011. A parallel SPH implementation on multi-core CPUs. Computer Graphics Forum 30, 1, 99-112.

Ihmsen, M., Cornelis, J., Solenthaler, B., Horvath, C., AND TESCHNER, M. 2013. Implicit incompressible SPH. IEEE Transactions on Visualization and Computer Graphics 99, PrePrints, 1.

JONES, J. 1924. On the determination of molecular fields. ii. from the equation of state of a gas. Proceedings of the Royal Society of London. Series A, Containing Papers of a Mathematical and Physical Character 106, 738, 463-477.

KAng, M., Fedkiw, R. P., AND LiU, X.-D. 2000. A boundary condition capturing method for multiphase incompressible flow. Journal of Scientific Computing 15, 3, 323-360.

Losasso, F., Gibou, F., AND FedKiw, R. 2004. Simulating water and smoke with an octree data structure. ACM Trans. on Graphics (SIGGRAPH Proc.), 457-462.

Macklin, M., AND Mueller, M. 2013. Position Based Fluids. ACM Trans. on Graphics (SIGGRAPH Proc.), To appear.

Misztal, M. K., Bridson, R., Erleben, K., Bærentzen, J. A., AND Anton, F. 2010. Optimization-based fluid simulation on unstructured meshes. Proceedings of Virtual Reality Interaction and Physical Simulation, VRIPHYS.

Monaghan, J. 2000. SPH without a tensile instability. Journal of Computational Physics 159, 2, 290-311.

Monaghan, J. 2005. Smoothed particle hydrodynamics. Reports on Progress in Physics 68, 8, 1703-1759.

MORRIS, J. 1999. Simulating surface tension with smoothed particle hydrodynamics. Int. J. Numer. Meth. Fluids 33, 3, 333-353.

Müller, M., Charypar, D., And Gross, M. 2003. Particlebased fluid simulation for interactive applications. In Proc. of the 2003 ACM SIGGRAPH/Eurographics Symposium on Computer Animation, 154-159.

NVIDIA ARC, 2011. mental ray 3.9 [software]. http://www.nvidia-arc.com/products/nvidia-mental-ray.html.
Runguiratananon, W., Kanamori, Y., And Nishita, T. 2012. Wetting effects in hair simulation. In Computer Graphics Forum, vol. 31, Wiley Online Library, 1993-2002.

Schechter, H., And Bridson, R. 2012. Ghost SPH for animating water. ACM Trans. on Graphics (SIGGRAPH Proc.) 31, $61: 1-61: 8$.

Shapiro, P. R., Martel, H., Villumisen, J. V., AND OWEN, J. M. 1996. Adaptive smoothed particle hydrodynamics, with application to cosmology: methodology. The Astrophysical Journal Supplement Series 103, 269.

SHEPARD, D. 1968. A two-dimensional interpolation function for irregularly spaced points. In Proceedings of the $23 \mathrm{rd}$ ACM national conference, 517-524.

Solenthaler, B., And Pajarola, R. 2009. Predictivecorrective incompressible SPH. ACM Trans. on Graphics (SIGGRAPH Proc.) 28, 3, 1-6.

Steele, K., Cline, D., Egbert, P. K., And Dinerstein, J. 2004. Modeling and rendering viscous liquids. Computer Animation and Virtual Worlds 15, 3-4, 183-192.

Takahashi, T., AND Fujishiro, I. 2012. Particle-based simulation of snow trampling taking sintering effect into account. In ACM SIGGRAPH 2012 Posters, ACM, 7.

Tartakovsky, A., And Meakin, P. 2005. Modeling of surface tension and contact angles with smoothed particle hydrodynamics. Physical Review E 72, 2, 26301.

Thürey, N., Wojtan, C., Gross, M., And Turk, G. 2010. A multiscale approach to mesh-based surface tension flows. $A C M$ Trans. on Graphics (SIGGRAPH Proc.) 29, 4, 48.

Yu, J., Wojtan, C., Turk, G., And Yap, C. 2012. Explicit mesh surfaces for particle based fluids. In Computer Graphics Forum (Eurographics Proc.), vol. 31, Wiley Online Library, 815-824. 